\title{
Cosmological simulations of the Milky Way
}

\section{Lucio Mayer}

Institute for Theoretical Physics, University of Zurich, Wintherthurestrasse 190, Zurich (CH)

\begin{abstract}
Recent simulations of forming low-mass galaxies suggests a strategy for obtaining realistic models of galaxies like the Milky-Way.
\end{abstract}

Cosmological simulations of galaxy formation are powerful tools for confronting the $\Lambda \mathrm{CDM}$ model with observational datasets. The increase in mass and spatial resolution and the improvement of sub-grid algorithms for star formation and feedback processes have recently resulted in simulated galaxies with realistic disk size and angular momentum content (Mayer et al. 2008; Governato et al. 2009a). However, simulated galaxies hosted in halos with masses $\sim 10^{12} M_{\odot}$ exhibit prominent bulges and structural parameters reminiscent of Sa spirals rather than of Sb/Sc galaxies. Surface densities at the solar radius are larger than that of the Milky Way (MW) by factors of a few and the more massive bulge produces a steeper rotation curve compared to that of the MW (Read et al. 2009). At halo masses $M_{\mathrm{vir}}>2 \times 10^{12} M_{\odot}$ the predominance of hot-mode gas accretion counters the presence of a prominent star forming disk at $z=0$, producing earlier-type objects resembling S0 galaxies (Brooks et al. 2009) and supporting recent estimates based on RAVE that yield $M_{\mathrm{vir}} \sim 10^{12} M_{\odot}$ (Smith et al. 2007).

Yet the solution to forming a realistic MW analog could be at hand. Recently we have performed galaxy-formation simulations for mass scales $<10^{11} M_{\odot}$. By sampling these low-mass galaxies with several millions of particles, we achieve a mass resolution better than $10^{3} M_{\odot}$ in the baryons, thus resolving individual molecular clouds. Star formation can now be tied to gas at molecular cloud densities $\left(\rho>100 \mathrm{~cm}^{-3}\right)$. A realistic, inhomogeneous interstellar medium is obtained that results naturally in stronger supernova outflows than when the standard star formation threshold $\left(\rho=0.1 \mathrm{~cm}^{-3}\right)$ is adopted. Such outflows efficiently remove the low-angular momentum baryonic material from the central region, suppressing the formation of a bulge and producing an object with a slowly rising rotation curve in very close agreement with observed dwarf galaxies (Governato et al. 2009b; see also Ceverino \& Klypin 2009). We argue that comparable resolution of MW-sized galaxies will yield rotation curves and bulge-to-disk ratios appropriate for $\mathrm{Sb}-\mathrm{Sc}$ spirals at $z=0$. This requires increasing the number of particles employed by more than an order of magnitude.

\section{References}

Brooks, A. M., Governato, F., Quinn, T., Brook, C. B., \& Wadsley, J., 2009, ApJ, 694, 396

Ceverino, D. \& Klypin, A., 2009, ApJ, 895, 292

Governato, F, Brook, C. B., Brooks, A. M., Mayer, L., Willman, B., Jonsson, P., Stilp, A. M., Pope, L., Christensen, C., Wadsley, J., \& Quinn, T., 2009a, MNRAS, 398, 312

Governato, F, Brook, C. B., Brooks, A. M., Mayer, L., Willman, B., Jonsson, P., Stilp, A. M., Pope, L., Christensen, C., Wadsley, J., \& Quinn, T., 2009b, Nature in press (arXiv:0911.2237)

Mayer, L., Governato, F., \& Kaufmann, T., 2008, Adv. Sci. Lett., 1, 7

Read, J., Mayer, L., Brooks, A.M., Governato, F., \& Lake, G., 2009, MNRAS, 397, 44

Smith, A., et al., 2007, MNRAS, 379, 755 